\title{
Mitteilungen der Deutschen Gesellschaft für Epileptologie e. V.
}

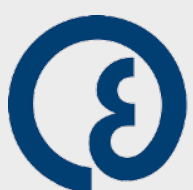

Deutsche Gesellschaft für Epileptologie e.v.

Deutsche Sektion der Internationalen Liga gegen Epilepsie

\section{Vorsitzender}

Prof. Dr. Andreas Schulze-Bonhage Epilepsiezentrum, Universitätsklinikum Freiburg Breisacher Str. 64, 79106 Freiburg Tel. 076127053660 Fax 076127050030 andreas.schulze-bonhage@ uniklinik-freiburg.de

\section{Vorsitzender}

Prof. Dr. med. Johannes Lemke Institut für Humangenetik Universitätsklinikum Leipzig AöR Philipp-Rosenthal-Str. 55, 04103 Leipzig Tel.: +49 3419723800

Fax: +493419723819 johannes.lemke@ medizin.uni-leipzig.de

\section{Geschäftsführerin}

Prof. Dr. Susanne Knake Leiterin Epilepsiezentrum Hessen Klinik für Neurologie Universitätsklinikum Marburg Baldingerstraße 35033 Marburg Tel: +49 (0)6421 5865435 Fax: +49 (0)6421 585444 Knake@med.uni-marburg.de

\section{Geschäftsführer}

Prof. Dr. med. Martin Holtkamp Epilepsie-Zentrum Berlin-Brandenburg Herzbergstr. 79, 10365 Berlin Tel. 030/5472-3501 Fax 030/5472-3502 martin.holtkamp@charite.de
Schatzmeisterin

PD Dr. med. Sarah von Spiczak

Klinik für Neuropädiatrie Norddt. Epilepsiezentrum Henry-Dunant-Str. 6 - 8 24223 Schwentinental Tel: 04307-909201

Fax: 04307-909260 s.spiczak@drk-sutz.de

\section{ILAE-Delegierter}

Prof. Dr. Andreas Schulze-Bonhage Epilepsiezentrum, Universitätsklinikum Freiburg Breisacher Str. 64, 79106 Freiburg Tel. 076127053660 Fax 076127050030 andreas.schulze-bonhage@ uniklinikfreiburg.de

\section{Geschäftsstelle}

Dt. Ges. für Epileptologie,

Petra Gehle

Reinhardtstr. 27c, 10117 Berlin

Tel. 0700/13141300

Fax 0700/13141399

office@dgfe.info

www.dgfe.info
Z. Epileptol. 2020 · 33:179

https://doi.org/10.1007/s10309-020 00329-5

(c) Springer Medizin Verlag GmbH, ein Teil von Springer Nature 2020

\author{
Herausgeber der Zeitschrift \\ für Epileptologie \\ Priv.-Doz. Dr. Friedhelm C. Schmitt \\ Universitätsklinik für Neurologie \\ Leipziger Str. 44, 39120 Magdeburg \\ Tel. 0391-67-14268 \\ Fax 0391-67-15216 \\ fc.schmitt@med.ovgu.de \\ Prof. Dr. Adam Strzelczyk, MHBA \\ Epilepsiezentrum Frankfurt \\ Rhein-Main \\ Universitätsklinikum Frankfurt \\ Schleusenweg 2-16, 60528 Frankfurt \\ Tel. $0696301-7466$ \\ Fax: 069 6301-84466 \\ strzelczyk@med.uni-frankfurt.de
}

\section{Neuer Termin der DGfE- Jahrestagung}

Wegen der Covid-19-Krise wird die Jahrestagung voraussichtlich auf den 6.-09.09.2020 in Freiburg verlegt.

Die Mitgliederversammlung wird sich dementsprechend verschieben. Wir informieren Sie rechtzeitig.

\section{Ergänzung der Geschäftsstelle Yvonne Jäger-Kolling}

Frau Gehle reduziert ihre Tätigkeit ab April. Frau Yvonne Jäger-Kolling aus dem Epilepsiezentrum Hessen, Uniklinik Marburg, wird die Geschäftsstelle zusammen mit Frau Gehle betreuen. Damit ist auch die Digitalisierung der Mitgliederverwaltung verbunden, die eine Reduzierung der Verwaltungstätigkeit/der Kosten verspricht.

\section{Neuer Studiengang in Ravensburg ab Oktober}

Das Zentrum für Psychiatrie Südwürttemberg (https://www.epilepsiezentrumbodensee.de) bietet Plätze für ein Duales Studium Medizintechnische Wissenschaften an. Dr. Hartmut Baier übt bei der Dualen Hochschule in diesem Bereich eine Lehrtätigkeit aus und kooperiert mit der Dualen Hochschule Heidenheim für diesen Studiengang. $\mathrm{Zu}-$ gangsvoraussetzung ist die Fachhochschulreife und abgeschlossene MTA-Ausbildung. Bei mittlerer Reife gibt es nach 2 Jahren Berufserfahrung die Möglichkeit einer Aufnahmeprüfung. Bewerbung bis 25.05.2020 Bewerberportal: www.zfp-karriere.de.

Nähere Auskünfte erteilt Ihnen gerne Dr. Hartmut Baier, Chefarzt der Abteilung Epileptologie Ravensburg/Weissenau, Tel.: 0751 76012390 (Hartmut.Baier@zfp-zentrum.de). 